\title{
Conceptual Behavior in Young Children: Learning to Shift Dimensional Attention ${ }^{1}$
}

\author{
DONNA J. SCHELL \\ linizeraty of Michant
}

\begin{abstract}
Four- and 5-year-old preschool children wore trained $(a)$ to identify the relevant unidimensional concept in a card sorting task and $(b)$ to shift quickly from concept to concept when the relevant dimension changed. The stimuli varied on three dimensions (shape, color, and quantity). Experimental conditions included Initial Test-Training, Shift Testing, and Special Training to establish unidimensional control and/or quick shifts in unidimensional control. Sperial Training included vorbal and nonverbal prompts. Trained children reached critcrion significantly faster than did control children on Final Testing, regardless of which dimension was relevant. This and other results support the conclusion that the experimental conditions contained rritical components of experience leading to a bohtrior which otherwise would not be observed in 4-and 5-rear-old children. The critical components of experience appear to be $(a)$ thit the children larn to attend to one dimension at a time when the stimuli vay on several dimensions and $(b)$ that they learn to shift quickly when required from dimension to dimension of a single set of multidimensional stimuli.
\end{abstract}

Previous studies have found that four- and five-year-old children are not likely to be successful on a task in which they must respond on the basis of a single dimension when the stimuli vary on several dimensions (Ginsberg, 1969; Inhelder \& Piaget, 1964; Nitler \& Harris, 1969; Osler \& Kofsky, 1965, 1966; Rieber, 1969). Osler and Kofsky $(1965,1966)$ have attributed the children's failure to their perseverating on irrelevant stimulus dimensions and inability to see more than one way of classifying the stimuli. Inhelder and Piaget (1964) have argued that the children do not yet have the cognitive capacity to change classification criteria. Mitler and Harris (1969) have suggested that suecess or failure is a

\footnotetext{
${ }^{1}$ Based on a dissertation submitted in partial fulfillment of the requirements for a Ph.D., University of Michigan. I wish to express my appreciation to the members of my dissertation committec. Pspecially to Darid Birch and to Edward L. Walker. William C. Stebbins. and Edwin J. Thomas. In addition. Robert Schell deserves extra special thanks for his indisponsable, personal encouragement and technical assistanec. This research was supported by a dissertation research grant from Ilorace H. Rackham School of Graduate Studies, the University of Michigan; and by a Rackham Prize Fellowship, funded by the Ford Foundation.
} 
matter of whether the relevant dimension is available and readily usable for any given child.

Saying that the children perseverate or are unable to change criteria does not explain the failing behavior, it only redescribes it. Saying that the relevant dimension is not available or is not readily usable is a more satisfactory explanation, but still the real question is what determines whether a dimension is available and readily usable. The present investigation went several steps further than previous studies by trying to identify the actual stimulus and response events which lead to success on conceptual tasks calling for unidimensional attention. More specifically it examined the experimental development of responding under the control of single dimensions of geometric stimuli and of quick shifts in responding from one dimension to another.

Numerous training studies have covered a variety of complex conceptual behaviors involving unidimensional control (e.g., Bijou, 1968; Caron, 1968; Gelman, 1969; Ginsberg, 1969; Saravo \& Kolodny, 1969; Tighe \& Tighe, 1969). However, only one of the studies which dealt with the same or nearly the same behavior that is of concern here, provided an effective training history (Suppes \& Rosenthal-Hill, 1968). Unfortunately, in that study, sample stimulus carls were always present, and these cards eventually came to serve as nonverbal prompts which "told" a child what dimension was relcvant. A test was not carried out to see how the children would perform without such prompts. While studies by Kofsky (1967), Osler \& Scholnick (1968), and Scholnick, Osler, \& Katzenellenbogen (1968) also failed to design completely effective experimental histories, they have, together with previous studies, suggested a number of procedures to follow as well as to avoid in training young children to identify the relevant dimension of multidimensional stimuli. For one thing, the training stimuli in the present experiment were made to vary on three rather than just one dimension. For another, instead of bringing about responding to each dimension only once, this experiment brought about quick shifts in responding to one and then to another and then to another dimension with the same set of multidimensional stimuli.

The prosent experiment also examined the effects of a nonverbal modification of the stimuli themselves, a procedure which in previous studies had facilitated training by increasing the probability that any given subject would attend to one dimension rather than another, or others (e.g., Bem., 1967; Bijou, 1968; Caron, 1968; Sidman \& Stoddard, 1967; Terrace, 1963). In addition, it assessed the effectiveness of including verbal prompts which involved telling a child which dimension to attend to. It was hoped that the use of these instructional prompts 
would lead eventually to a child's prompting himself with such instructions (cf., Bireh, 1966; Lovaas, 1964; Luria, 1964).

\section{METHOD}

Subjects

The $S_{s}$ were children, predominantly from college-educated and professional families, attending preschools in Ann Arbor, Michigan. ${ }^{2}$ There were 36 experimental and 36 control $S$ s. The 36 experimental $S \mathrm{~s}$ were divided into three experimental treatment conditions, $12 \mathrm{Ss}$ in each condition. The children ranged in age from 4 years 0 months to 5 years 5 months with the mean age in each experimental and control condition 4 years 10 months.

\section{Apparatus}

\section{Manipulandum}

A "Talking Learning Machine" manufactured by Mattel, Inc., was the manipulandum. The machine was placed on a low table at which $S$ was seated. $E$ sat to $S$ 's right. When $S$ pushed on a small bar located at the front of the machine, a plastic card fell down from a hopper above to a position directly in S's view. Then depending on which stimulus was displayed on the face of the card, $S$ was either to push the card over to the left or over to the right along the front track of the machine. After each response, $S$ handed the card to $E$ who placed it out of $S$ 's view.

\section{Discriminative Stimuli}

The training stimuli (Set 1) varied with regard to two attributes along three dimensions, specifically the following: shape (square or circle), color (red or blue), and quantity (onc or two). The forms were outlincd in black ink, cut out, and pasted on white plastic cards. In addition to this set of eight stimuli, $S \mathrm{~s}$ in the Nonverbal condition were presented in the first stage of training wilh three other sets of eight geometric stimuli. In each set the eight stimuli were comprised of the same orthogonal combinations of the two attributes of the above mentioned dimensions but the stimuli were modified as follows. For shape relevant

2I am grateful to the teachers and the following directors of preschools in Ann Arbor, Michigan: Mrs. Mary Burns, Iittle Farm Nursery School; Mrs. Michelle Pesek, The Gay-Jay Nursery School; Mrs. Elizabeth McHale, Perry Nursery School: Mrs. Virginia Otto. Tittle Lamb Day Nursery. Their helpful cooperation was an important contribution to this and an earlier pilot investigation. 
the colors were faded, i.e., of low saturation (Set 2). For color relevant the forms were outlined with dots (Set 3). For quantity relevant the colors were faded and the forms were outlined with dots (Set 4). The testing stimuli varied on the same dimensions as the training stimuli, but the attributes of the dimensions were different: shape (triangle or rectangle), color (green or yellow), and quantity (three or four).

\section{Procedure}

\section{Phase I: Familiarization}

Phase I acquainted $S$ with the apparatus and the general experimental procedure. The $S$ had to discover, without $E$ telling him, that a card picturing a bird was to be pushed to one side and a card picturing a cat to the other side. In a predetermined random order the cards were presented one at a time until $S$ responded correctly 12 consecutive times. After each of $S$ 's responses $E$ said either, "yes, good," or "no, look again at this picture-over this way" ( $E$ gesturing appropriately and then helping $S$ to correct his mistake).

In addition, plastic chips were dispensed on a fixed-ratio schedule which required a series of responses without intervening errors. The size of the ratio was increased or decreased depending on a S's success or failure in meeting the previous ratio requirement. Small pebbles were used to indicate how many consecutive correct responses $S$ had to make before he would receive a chip. At the end of each experimental session, $S$ exchanged the chips he had received for candy corn, M \& M's, peanuts, and/or miniature marshmallows. This reinforcement procedure was used during all phases (see Schell, 1969, for a more detailed description).

\section{Phase II: Training}

Training was divided into four parts: (1) Initial Test-Training, (2) Special Training: Unidimensional Control, (3) Shift Testing, and (4) Special Training: Shifts in Unidimensional Control. There were three experimental conditions, but they differed from each other in Initial TestTraining only.

Initial Test-Training-Basic condition. Following Familiarization, $E$ said:

Now I have some new pictures for you. [ $E$ put in the hopper the complete Set 1 (all eight cards picturing the geometric stimuli) plus one blank card on top.] Some of the pictures go over this way and some of the pictures go over this way $[E$ gesturing appropriately]. Go ahead and press down on the bar. Look at the picture. I'll help you do just the first one. 
The stimuli were placed in the hopper in blocks of eight until si responded rorrectly 16 conserentive times, or until ti4 trials had been completed, with one particular dimension relevant. On an arbitrary hasis, shape was relevant first, then color, then quantity. The maximum time for any given training session was 30 min. Within that limit $S$ continued to the next relevant dimension if he reached criterion on the prior one. If $S$ did not reach riterion with a particular dimension relevant, he still went on - in the next training session-to the next dimension relevant.

Whenever there was a change in the relevant dimension, $E$ said:

Now you'll play the game the same way. Press down on the bar. Look at the picture, and then mush the pieture over this way or over this way. But you'll see that there's a change in the game now. Go ahead and press down on the bar. I'll help you do the first one.

Initial Test-Training in the Verbal condition was the same as Initial Test-Training in the Basic condition in every regard except the following: For the initial training with each dimension relevant, just after $S$ pushed the bar bringing the picture down to its resting position on the track, $E$ said, "Look at the shape of the things in the picture" (or "Took at the color of the things in the picture" or "Look at how many things are in the picture"). The $E$ made these statements on every trial until $S$ responded correctly four consecutive times. Then beginning with the next block of trials, the number of times these verbal prompts were presented was decreased geometrically to zero. Trials continued until s had responded 16 consecutive times without the verbal prompts. If $S$ never made a string of 4 correct responses, the maximum number of prompts he could receive was 64 ; this because the maximum number of trials was 64.

Initial Test-Training in the Nonverbal condition was the same as Initial Test-Training in the Basic condition in every regard except the following: When shape was relevant, the modified stimuli of Set 2 were presented; when color was relevant, the modified stimuli of Set 3 were presented; and when quantity was relevant, the modified stimuli of set 4 were presented. For each set the modified stimuli were presented on every trial until $S$ responded correctly four consecutive times. Then they wore presented intermittently, intermixed with the stimuli of Set 1 . The modified stimuli then were presented in a decreasing fashion just as the verbal prompts were presented in the Verbal condition. Trials continued until $S$ had responded correctly 16 consecutive times with stimuli from Set 1 only. Just as in the Basic and the Verbal conditions, the criterion was standardized; 16 consecutive correct, 64 trials the limit for any dimension relevant, and $30 \mathrm{~min}$. the limit for session length. 
Special Training to establish unidimensional control was instituted with any $S$ who did not reach criterion with at least two of the three dimensions relevant in Initial Test-Training. This training included presentation of the verbal prompts just as in the Verbal condition. If this procedure did not work, then $E$ showed the child the correct sort and stated the rule for the sort (Bem, 1968; Blank, 1966; Suppes \& RosenthalHill, 1968).

Shift Testing took place during the first 32 trials in which a particular dimension was relevant after completion of Initial Test-Training and Special Training, if Special Training had been necessary for any given $S$. Only the regular, unmodified geometric stimuli were presented. With each change in the relevant dimension, $E$ said only, "Now there's going to be a change in the gane." The order in which the dimensions were relevant was arranged so as (1) to be different from the just preceding order, which for some $S$ s included the order in which the dimensions were relevant in Special Training, and (2) so that each of the three dimensions might be relevant in the same session.

Special Training to establish quick shifts in control was instituted with any $S$ who did not reach criterion on Shift Testing, i.e., did not reach criterion on all three dimensions, each within 32 trials. Besides the previously-mentioned special training procedures, this training included verbal prompts, e.g., "Look at the shape," said once, and nonverbal prompts. The nonverbal prompts were pairs of stimulus cards which varied only with regard to the relevant dimension. For example, if $E$ wanted to give $S$ a clue that color was relevant, she showed him a blue circle going one way and a red circle going the other way (Suppes \& Rosenthal-Hill, 1968). This was an abbreviated form of the special training to establish unidimensional control in which the $E$ showed $S$ the complete sort and stated the rule for the sort. Shift Training sessions continued until $S$ reached criterion with a shift to each of the relevant dimensions, within one session, and with no special training on any of the dimensions. (See Fig. 1 for a flow chart of alternative procedures followed with $S$ s who did and $S$ s who did not reach criterion in each subphase of training).

\section{Phase III: Final Testing}

After shifts in control to each of the three relevant dimensions had been established in Phase II ('Training), $S \mathrm{~s}$ in the Basic, Verbal, and Nonverbal conditions proceeded in the next session to Phase III (Final Testing). $S \mathrm{~s}$ in the Control condition proceeded directly from Phase I (Familiarization) to Phase III (Final Testing).

At the beginning of Final Testing $E$ said: 


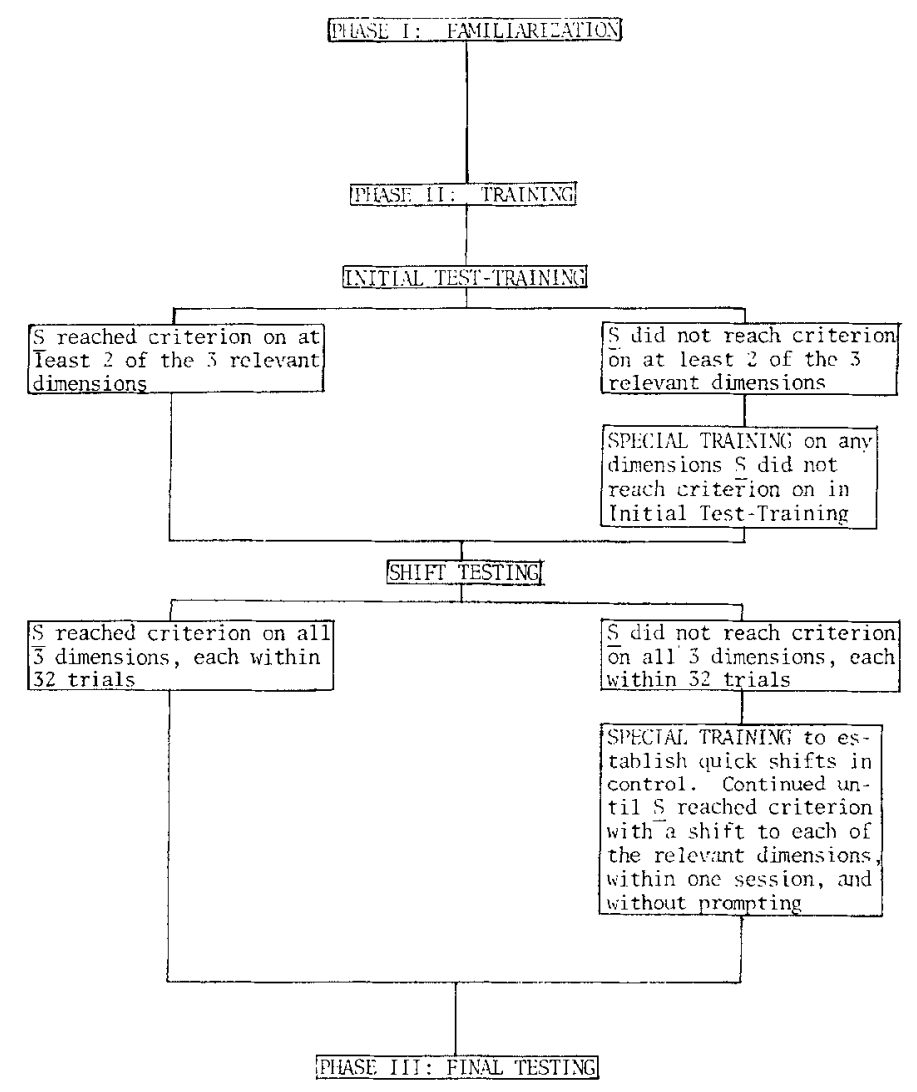

FIG. 1. Procedural sequences for expcrimental subjects.

Now you're going to play the same game, but the pictures will be new to you. Some of the pictures go over this way and some of the pictures go over this way. Go ahead and push on the bar. Look at the picture. I'll help you do just the first one.

The testing stimuli wcre presentcd until $S$ responded correctly 16 consecutive times within 144 trials. For each $S$, only one dimension was ever relevant in Final Testing. Ss were assigned a relevant dimension so that: (1) One-third $S$ s from each condition were assigned to shape, onethird to color, and one-third to quantity, (2) the mean age for Ss with each dimension relevant remained close to the grand mean, i.e., 4 years 10 months, and (3) any given experimental $S$ was assigned a dimension that was not the last one he had relevant in training.

\section{RESULTS AND DISCUSSION}

A preliminary statistical analysis revealed no significant Sex or Age effects on either Familiarization, Initial Test-Training, or Final Testing 
trials to criterion. Therefore, in all subsequent analyses the data were collapsed across these factors.

\section{Familiarization}

For the Verbal condition the mean number of trials to criterion on the Familiarization task was 17.6, for the Nonverbal 21.5, for the Basic 19.2, and for the Control condition 21.1. There were no significant differences among these mean values $(F=.610, d f=3,68)$.

\section{Initial Test-Training}

Figure 2 illustrates the mean trials-to-criterion results for Conditions across Dimensions in Initial Test-Training. In general, $S_{s}$ in the Verbal condition did better than $S \mathrm{~s}$ in either the Nonverbal or Basic conditions. Also Ss did better on Shape than on Color, and better on Shape than on Quantity. For the Verbal condition the mean number of trials to criterion was 33.4 on Initial Test-Training, 50.7 for Nonverbal, and 55.6 for Basic. For the Shape dimension the mean number of trials to criterion on Initial Test-Training was 40.0, for Color dimension 50.3, and for the Quantity dimension 49.3.

The results of a $3 \times 3$ (Experimental Conditions $\times$ Dimensions) mixed design analysis of variance of trials to criterion revealed a significant main effect of Experimental Conditions $(F=21.56, d f=2,33, p<.005)$ and Dimensions $(F=6.97, d f=2,66, p<.005)$. The interaction effect was not significant $(F=2.08, d f=4,66$ ). (It can be seen in Fig. 2 that there is only one point which does not fit the main effects trends: that is the mean trials to criterion for the Nonverbal condition when Shape was the relevant dimension.)

Post hoc analysis of the Experimental Conditions main effect (Significant Critical Difference $=8.77, p<.05$, Tukey [a] test) showed that $S \mathrm{~s}$ in the Verbal condition reached criterion significantly faster than did $S s$ in the Nonverbal condition and significantly faster than did $S$ s in the

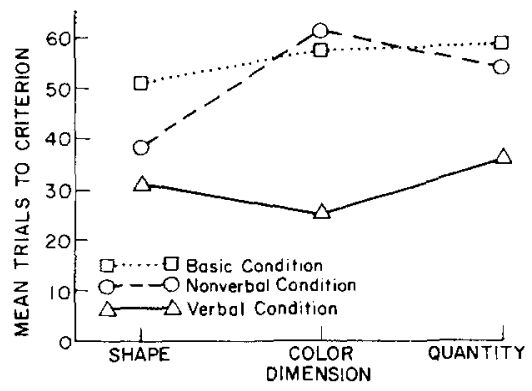

FIG. 2. Mean trials to criterion for each experimental condition on each dimension on Initial Test-Training. 
Basic condition; the difference between the Xonverbal and Basic conditions was not significunt. Post hoc analysis of the Joinensions main effect (Significant Critical Difference $=7.33, p<05$, Tukey lal test.) showed that significantly fewer trials were required to reach eriterion on Shape than on Color, and fewer on Shape than on Quantity.

It is apparent that telling the children which dimension to look at (i.e., the Verbal condition) worked best to bring about correct responding rapidly. The rerbal instruction was like a prior hint to the rhild as to which response he should make (response prompting). In addition, after the child made the response, $E$ indicated to him whether the response was right or wrong (response confirmation). In the Basic condition $E$ just indicated right or wrong after a response was made. The finding of superiority of the Verbal condition over the Basic condition agrees then with findings that response prompting procedures equal or surpass the effectiveness of response confirmation procedures and that a combination of the two procedures promises to be most effective (see review by Aiken \& Iau, 1967).

The Basic condition repeated the expcrimental conditions of previous studies of this same conceptual behavior in preschool children who had no prior experimental training. There was no significant difference in performance when any of the three dimensions was relevant, just as was the case for children in the studies by Osler and Kofsky (1965, 1966), and for control children in experimental-training studies (Kofsky, 1967; Osler \& Scholnick, 1968; Scholnick, Osler, \& Katzenellenbogen, 1968). This trend in results held true even though the previous studies included size as a relevant dimension (plus shape and/or color) whereas this study included quantity (plus shape and rolor).

\section{Special Training: Unidimensional Control}

No special training to establish unidimensional control was required for children in the Verbal condition. For children in the Nonverbal condition who had to have special training, it took an average of 1.1 additional training sessions to establish control by Color and an average of 1.1 additional training sessions to establish control by Quantity. There was no significant difference in the average number of spccial training trials required to reach criterion with Color and Quantity.

For children in the Basic condition who had to have special training, control was established by each dimension (Shave, Color, and Quantity) in an average of from 1.0 to 1.5 additional sessions. Significantly fewer trials of special training were required to establish control by the Quantity dimension than by either the Shape or Color dimensions. However, further examination of the raw data showed that this finding was due 
to whether Ss had previous special training on both Shape and Color. Only those Ss who had previous training on Shape and Color reached criterion significantly faster under special training on Quantity.

There was no significant difference between the Nonverbal and the Basic conditions in the number of special training trials required to reach criterion on Color and on Quantity. Thus it appears that the previoui Initial Test-Training in the Nonverbal and Basic conditions on color and quantity neither interfered with nor particularly hastened the establishment of control with the special training. That special training was sufficient for both the Nonverbal and the Basic groups perhaps is not too surprising since it commenced with procedures identical to those previously used in Initial Test-Training in the Verbal condition.

\section{Shift Testing}

The overall Shift Testing performance of $S_{s}$ in each of the Experimental conditions was very nearly the same. However, within the Basic condition, Ss performed better on Color thin on Shape, and better on Color than on Quantity. The performance of $S \mathrm{~s}$ in the Verbal and in the Nonverbal condition was the same from dimension to dimension.

A $3 \times 3$ (Experimental Conditions $\times$ Dimensions) mixed design analysis of variance of trials to criterion revealed the Experimental Conditions effect was nonsignificant $(F<1, d f=2,33)$, as was the interaction effect $(F=1.85, d f=4,66)$. The main effect of Dimensions was significant $(F=6.99$, df $-2,66, p<.005)$. Further post hoc analysis showed that this effect was due mainly to the fact that significantly fewer trials were required by $S_{\mathrm{s}}$ in the Basic condition to reach eriterion on Color than on Shape or Quantity (see Table 1).

The purpose of Shift Testing was to determine whether one previous occasion in which control was established by each of the dimensions along which a set of stimuli vary would be sufficient to assure that a quick shift in unidimensional control would oceur on a subsequent occasion.

TABLE: 1

Condition $\times$ Dimengion Mfans for Trills to Chiterion: Shift Testing

\begin{tabular}{lccc}
\hline & & \multicolumn{3}{c}{ Condilion } \\
\cline { 3 - 4 } Dimension & Verbal & Nonverbal & Basic \\
\hline Shape & 30.17 & 30.17 & $30.17^{a}$ \\
Color & 27.17 & 28.92 & $23.83^{a, b}$ \\
Quantity & 28.75 & 29.33 & $29.92^{b}$ \\
\hline
\end{tabular}

${ }^{a, h}$ Vertical entries with the same adjacent superscript are significantly different at $p<.05$; by Tukey (a) test, Significant Critical Difference $=4.10$. 
Clearly, it was not sufficient. And this held regardless of whether a child was in the Verbal, the Nonverbal, or the Basic condition; whether a child had special training; and whether control was eventually established in special training by means of the verbal prompting or the sorting procedure.

It was only in the Basic condition that Shift Testing performance differed depending on which dimension was relevant. Just why more of the children in the Basic condition reached criterion, and did so in fewer trials, on shift to color than to shape or quantity remains uncertain. Inspection revealed no particular personal characteristic(s) or training procedure feature that would appear to account for these results.

Most of the experimental $S$ s were not successful on Shift Testing: 25 out of 36. Twenty out of the 25 had experimental histories which included verbal prompts; a few more had the sorting procedure in addition to the verbal prompts. These conditions were effective in training the children to attend to each of the dimensions of the stimuli in turn. However, when these extra helps were not given, the children did not shift on their own from one dimension to another. At least they did not do so within the relatively few, 32 trials allowed for each shift. In general, it is concluded that special training to establish unidimensional control did not significantly improve performance on a subsequent task which required a quick shift in unidimensional control.

Special Training: Shifts in Unidimensional Control

For $S$ s who were not successful on Shift Testing the average number of sessions required to establish quick shifts in control to Shape, Color, and Quantity, respectively, was 2.6, 2.2, and 2.6 for $S \mathrm{~s}$ in the Verbal condition, 3.0, 3.4, and 2.6 for $S \mathrm{~s}$ in the Nonverbal condition, and 3.1, 2.5, and 3.0 for $S \mathrm{~s}$ in the Basic condition. In both the Verbal and the Nonverbal condition there was no significant difference in the number of special training trials required to establish quick shifts in control to Shape, Color, or Quantity. Within the Basic condition, significantly fewer training trials were required to establish a quick shift in control to Color than to either Shape or Quantity. However, there were no significant differences among the experimental conditions in the average number of training trials required to establish quick shifts in control to either Color, Shape, or Quantity. Furthermore, the verbal prompts were sufficient $93 \%$ of the time to establish control in any particular instance of special training.

What at first stands out here is that if a child needed special training on shifting, then the same amount was required regardless of whether he had had the Verbal, the Nonverbal, or the Basic experimental training 
initially. However, this finding is not surprising considering that the differences between the experimental conditions had been diminished by the special training to establish unidimensional control. Furthermore, special training to establish quick shifts in unidimensional control eliminated any differences in experimental histories for most of the children.

One of the most important conclusions to be drawn, is that speaking of most 4- and 5-year-old children on this particular concept task, there must be many training trials before a child will shift quickly from concept to concept as the relevant dimension changes. Furthermore, there must be many occasions on which the child is aided by a verbal or nonverbal prompt before he will shift quickly when necessary, on his own.

\section{Final Testing}

The final analysis was of the trials-to-criterion data of Final Testing. Because of heterogeneity of variance $\left(\chi^{2}=83.10, d f=5, p<.01\right.$, Bartlett's Test) and some skewness, a logarithmic transformation was performed on the trials-to-criterion scores. This transformation failed to reduce the variance differences sufficiently $\left(\chi^{2}-82.89, d f=5, p<.01\right.$, Bartlett's Test). Therefore a reciprocal transformation was performed. A Bartlett's Test on this transformed trials-to-criterion data indicated no significant variance differences $\left(\chi^{2}=9.71, d f=5\right)$.

$S_{\mathrm{s}}$ in the original three Experimental conditions performed alike on Final Testing. Further, these $S \mathrm{~s}$, taken as a whole, performed better than $S_{s}$ in the Control condition, regardless of which was the relevant dimension. $S \mathrm{~s}$ in the Experimental condition performed the same on all three relevant dimensions, while $S_{s}$ in the Control condition performed better on Shape than on Quantity.

The transformed trials-to-criterion data were analyzed in two steps: An initial fixed effects analysis of variance revealed no significant difference $(F<1, d f=2,27)$ among the three Experimental conditions in the number of trials required to reach criterion on Final Testing. Neither was there a significant Dimensions $(F=2.996, d f=2,27)$ nor interaction $(F<1, d f=4,27)$ effect. For purposes of subsequent analysis and comparison with the Control condition, the three Experimental conditions were combined to form one Experimental condition.

A $2 \times 3$ (Conditions $\times$ Dimensions) fixed effects analysis of variance of trials to criterion revealed significant Conditions $(F=39.42, d f=1,66$, $p<.005)$ and a significant Dimensions $(F==7.24, d f=2,66, p<.005)$ main effect. The interaction was not significant $(F<1, d f=2,66)$. Analysis of the Conditions effect showed that the Experimental $(\bar{X}$ trials $=$ 23.39) condition $S s$ reached criterion in significantly fewer trials than $\mathrm{Ss}$ in the Control $(\bar{X}$ trials $=84.11)$ condition $(F=39.42, d f=1,66$, 
TABLI: :

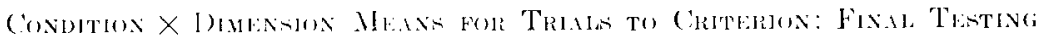

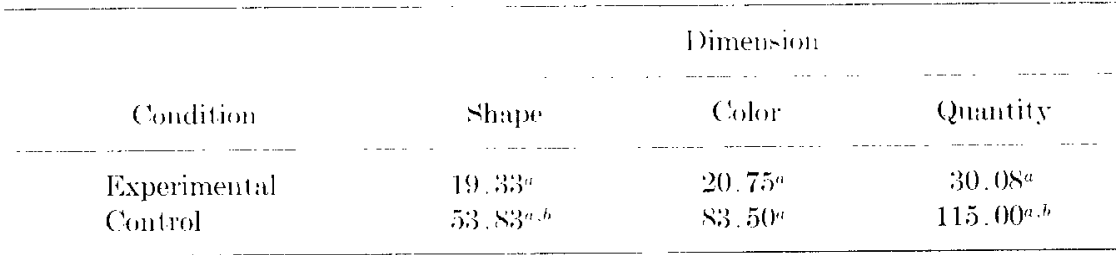

${ }^{a, b}$ Vertically adjacent (ondition entries with superseripi $a$ and horizontally aligned Dimension entries with superscript $b$ are significantly different at $p<.05$; by Critical Difference based on transformed data: Tukey (a) test.

$p<.005)$. In addition, as shown in Table 2, Ss in the Experimental condition reached criterion in significuntly fewer trials than those in the Control condition on all three dimensions.

Post hoc analysis of the Dimensions main effect (Significant Critical Difference for transformed data $=.01118, p<.05$, Tukey $[a]$ test) indicated that there wa: no significant difference in trials to criterion on Color $(\bar{X}-52.12$, or .03582 for the transformed scores) as compared to Shape $(\bar{X}:=36.58$, or .04503 for the transformed scores) or Quantity $(\bar{X}=72.54$, or .02736 for the transformed scores). However, significantly fewer trials were required to reach eriterion on Shape than on Quantity. As can be seen in Table 2, this significant difference is mainly due to the fact that the Control Ss reached criterion in significantly fewer trials on Shape than on Quantity.

The results decidedly favor those children who had experimental training, regardless of which dimension was relevant. (That there was no significant difference among the Final Testing performances of children in each of the original three experimental conditions is entirely understandable; by the end of training, most of the children's experimental historics contained all the same elements, excepting a few shildren who were given modified stimuli carly in training.) Thus, it appears that children with experimental training were more proficient on the task than were children lacking experimental training, and irrespective of which dimension was relevant.

Furthermore, it appears that the experimental training made it just as likely that any of the three dimensions would come to control the responding of the experimental children. The performance of the control group of children indicates that without such training the shape dimension was easier than the (quantity dimension. (On similar concept-identification tasks Mitler and Harris (1969) found shape to be easier than quantity for kindergarten children also.

The results of shift Testing in this experiment also must be kept in 
mind when the differences in the Final Testing performance of the experimental and control children are considered. Initial Test-Training (or Special Training to establish unidimensional control) brought responding under the control of each dimension in turn, and thereby presumably equalized the probabilities for particular dimensions coming to control responding. It leveled the "attending hierarchy," as Baron (1965) would say, or it made each dimension equally "available" (Mitler \& Harris, 1969). However, the Shift Testing results suggest that just equalizing the probabilities of any particular dimension coming to control responding will not assure that quick shifts in control to each dimension will take place on later occasions. Quick shifts in control also must be established. In Mitler and Harris' terms this would mean making all the dimensions readily "usable" for each child. It is concluded that, though the experimentally-trained children may have been just as likely to attend initially to any one of the dimensions of the testing stimuli, they quickly shifted their attention among the dimensions if such shifting was required. Most of the control children did not shift their dimensional attention at all, let alone shift it quickly.

\section{Concluding Remarks}

The conditions for the development of complex conceptual behavior in young children include the contingencies of stimulus and response events set up in the present experiment. Particularly, this experimental training led to the intended conceptual behavior where previous experimental training (Schnolnick \& Osler, 1968; Scholnick, Osler, \& Katzenellenbogen, 1968) did not because (1) the discriminative stimuli in the presenl sludy varied on several dimensions, and (2) quick shifts in control were established with the same set of multidimensional stimuli. The results indicate that the stimulus and response conditions contained in the experimental training are sufficient to develop the intended conceptual behavior. The results also indicate they are necessary prior conditions for this particular conceptual behavior insofar as they are contrasted with the conditions and results of previous training studies.

The broadest conclusion that may be drawn is that conceptual behavior is a function of training. This conclusion has the merit of being an independent, noncircular explanation for the behavior. And it suggests that success or failure on a conceptual task is not a function of the presence or absence of some cognitive capacity to carry out the task. Rather, success or failure is a function of having or not having the experience necessary to carry out the task.

This investigation also answered some questions about the possible facilitating effects of some verbal and nonverbal manipulations in the 
experimental development of conceptual behavior. Verbal prompts were most effective overall in hastening the establishment of unidimensional control and of quick shifts in control. The effects of the verbal prompts were immediate in the initial establishment of control by each of the dimensions along which the stimuli varied. This immediate effect suggests that verbal control by these particular instructions from an adult is already quite strong in 4- and 5-year-old preschool children. Evidence of control by these instructions then extends the generality of previous results which indicated that control by various other instructions from an adult is fairly strong with 4- and 5-year-old children (Birch, 1966; Lovaas, 1964; Luria, 1964; Meichenbaum \& Goodman, 1969). Nevertheless, the verbal prompts had to be presented on many occasions before a child would shift quickly without being prompted. It seems reasonable to assume that this is what happens in the real life development of the behavior.

\section{REFERENCES}

Aiken, E. G.. \& LaU, A. W. Response prompting and response confirmation: a review of recent literature. Psychological Bulletin, 1967, 68, 330-341.

Baron, M. R. The stimulus, stimulus control, and stimulus generalization. In D. I. Mostofsky (Ed.), Stimulus generalization. Stanford: Stanford Univ. Press. 1965. Pp. 62-71.

Bem. S. L. Verbal self-control: The establishment of effective self-instruction. Journal of Experimental Psychology, 1967. 74, 485-491.

BEM, S. L. The role of task comprehension in children's problem-solving. Unpublished doctoral dissertation, University of Michigan, 1968.

BıJou. S. W. Studies in the experimental development of left-right concepts in retarded children using fading techniques. In N. R. Ellis (Ed.), International review of research in mental retardation. Vol. 3. New York: Academic Press, 1968. Pp. 66-96.

Birch, D. Verbal control of nonverbal behavior. Journal of Experimental Child Psychology, 1966. 4, 266-275.

BLANK. M. The effects of training and verbalization on reversal and extradimensional learning. Joutral of Experimental Child Psychology, 1966, 4, 50-57.

Caron, A. J. Conceptual transfer in preverbal children as a eonsequence of dimensional training. Journal of Experimental Child Psychology, 1968, 6, 522-542.

Gelman, R. Conservation acquisition: a problem of learning to attend to relevant attributes. Journal of Experimental Child Psychology, 1969, 7, 167-187.

Ginsbera, R. Number and color responses in the young child. Journal of Experimental Child Psychology, 1969, 7, 265-273.

Inhelder, B., \& Piaget, J. The early growth of logic in the child. New York: Harper, 1964.

KoFsKY, E. The effect of verbal training on concept identification in disadvantaged children. Psychonomic Science, 1967, 7, 365-366.

LovaAs, O. I. Cue properties of words: the control of operant responding by rate and content of verbal operants. Child Development, 1964, 35, 245-256.

Luria, A. R. The development of the regulatory role of speech. In R. J. C. Harper, 
C. C. Anderson, C. M. Christensen, and S. M. Hunka (Eds.), The cognitive processes: Readings. Englewood Cliffs, N. J.: Prentice-Hall, 1964. Pp. 601-622.

Meichenbaum, D., \& Goodman, J. The developmental control of operant motor responding by verbal operants. Journal of Experimental Child Psychology, 1969, 7, 553-565.

Mitler, M. M., \& Hakis, L. Dimension preference and performance un a series of concept identification tasks in kindergarten, 1st grade, and 3rd grade children. Journal of Experimental Child Psychology, 1969, 7, 374-384.

OsLer, S. F., \& Kofsky, F. Stimulus uncertainty as a variable in the development of conceptual ability. Journal of Experimental Child Psychology, 1965, 2, 264279 .

Osler, S. F., \& KoFsky, E. Structure and strategy in concept learning. Journal of Experimental Child Psychology, 1966, 4, 198-209.

Osler, S. F., \& Scholnick, E. K. The effect of stimulus differentiation and inferential experience on concept attainment in disadvantaged children. Journal of Experimental Child Psychology, 1968, 6, 658-666.

Rieber, M. Hypothesis testing in children as a function of age. Developmental Psychology, 1969, 1, 389-395.

Saravo, A., \& Kolodny, M. Learning set and shift behavior in children. Journal of Experimental Child Psychology, 1969, 7, 21-30.

Schell, D. J. M. Development of unidimensional concept behavior in children: Verbal and nonverbal control. Unpublished doctoral dissertation, University of Michigan, 1969.

Scholnick, E. K.. Osler, S. F., \& Katzenellenbogen, R. Discrimination learning and concept identification in disadvantaged and middle class children. Child Development, 1968, 39, 15-26.

Sidman. M., \& Stoddard, L. T. The effectiveness of fading in programming a simultaneous form discrimination for retarded children. Joumal of the Experimental Analysis of Behavior. 1967, 10, 3-16.

Suppes, P., \& Rosentahl-Hill, I. Concept formation by kindergarten children in a card-sorting task. Journal of Experimental Child Psychology, 1968, 6, 212-230.

Terrace, H. S. Discrimination learning with and without "errors." Journal of the Experimental Analysis of Behavior, 1963, 6, 1-27.

Tiane, L. S., \& Tighe, T. J. Transfer from perceptual pretraining as a function of number of task dimensions. Journal of Experimental Child Psychology, 1969, $8,494-502$. 